\title{
Review Article \\ Left Atrial Appendage: Physiology, Pathology, and Role as a Therapeutic Target
}

\author{
Damiano Regazzoli, ${ }^{1}$ Francesco Ancona, ${ }^{1}$ Nicola Trevisi, ${ }^{2}$ Fabrizio Guarracini, ${ }^{2}$ \\ Andrea Radinovic, ${ }^{2}$ Michele Oppizzi, ${ }^{1}$ Eustachio Agricola, ${ }^{1}$ Alessandra Marzi, ${ }^{2}$ \\ Nicoleta Carmen Sora, ${ }^{2}$ Paolo Della Bella, ${ }^{2}$ and Patrizio Mazzone ${ }^{2}$ \\ ${ }^{1}$ Non-Invasive Cardiology, Cardio-Thoracic-Vascular Department, San Raffaele Scientific Institute, Via Olgettina 60, \\ 20132 Milano, Italy \\ ${ }^{2}$ Arrhythmology and Electrophysiology Unit, Cardio-Thoracic-Vascular Department, San Raffaele Scientific Institute, \\ Via Olgettina 60, 20132 Milano, Italy
}

Correspondence should be addressed to Damiano Regazzoli; regazzoli.damiano@hsr.it

Received 28 November 2014; Revised 22 January 2015; Accepted 25 January 2015

Academic Editor: Kimimasa Tobita

\begin{abstract}
Copyright (C) 2015 Damiano Regazzoli et al. This is an open access article distributed under the Creative Commons Attribution License, which permits unrestricted use, distribution, and reproduction in any medium, provided the original work is properly cited.
\end{abstract}

\begin{abstract}
Atrial fibrillation (AF) is the most common clinically relevant cardiac arrhythmia. AF poses patients at increased risk of thromboembolism, in particular ischemic stroke. The CHADS2 and CHA2DS2-VASc scores are useful in the assessment of thromboembolic risk in nonvalvular AF and are utilized in decision-making about treatment with oral anticoagulation (OAC). However, OAC is underutilized due to poor patient compliance and contraindications, especially major bleedings. The Virchow triad synthesizes the pathogenesis of thrombogenesis in AF: endocardial dysfunction, abnormal blood stasis, and altered hemostasis. This is especially prominent in the left atrial appendage (LAA), where the low flow reaches its minimum. The LAA is the remnant of the embryonic left atrium, with a complex and variable morphology predisposing to stasis, especially during AF. In patients with nonvalvular AF, $90 \%$ of thrombi are located in the LAA. So, left atrial appendage occlusion could be an interesting and effective procedure in thromboembolism prevention in AF. After exclusion of LAA as an embolic source, the remaining risk of thromboembolism does not longer justify the use of oral anticoagulants. Various surgical and catheter-based methods have been developed to exclude the LAA. This paper reviews the physiological and pathophysiological role of the LAA and catheter-based methods of LAA exclusion.
\end{abstract}

\section{Introduction}

The Left atrial appendage (LAA) has a complex anatomical structure that is distinct from the rest of the left atrium as it has different embryologic, anatomic, and pathophysiologic characteristics.

LAA is a remnant of the embryonic left atrium [1], while the rest of the left atrial cavity derives from an outgrowth of the pulmonary veins.

In order to define LAA anatomy and topographic relationships, multidetector computerized tomography (CT), and its high definition and transesophageal echocardiogram (TEE), in particular with the development of threedimensional reconstructions, are the most accurate noninvasive imaging modalities. Cardiac magnetic resonance imaging (MRI) is an emerging technique in order to detect thrombi as well as LAA sizing [2], but its use in the clinical setting remains limited mainly due to its high costs and poor temporal resolution.

LAA is not just an embryologic remnant, but it seems to play an important role in the regulation of heart rate and fluid balance [3].

On the other hand, LAA has a key role in the thromboembolic risk [4] associated with atrial fibrillation (AF) and it could also have a possible triggering effect of atrial tachyarrhythmias [5].

Because of this role in physiology and pathophysiology, LAA is recently gaining attention as a therapeutic target especially in thromboembolism prevention in patients with AF. 


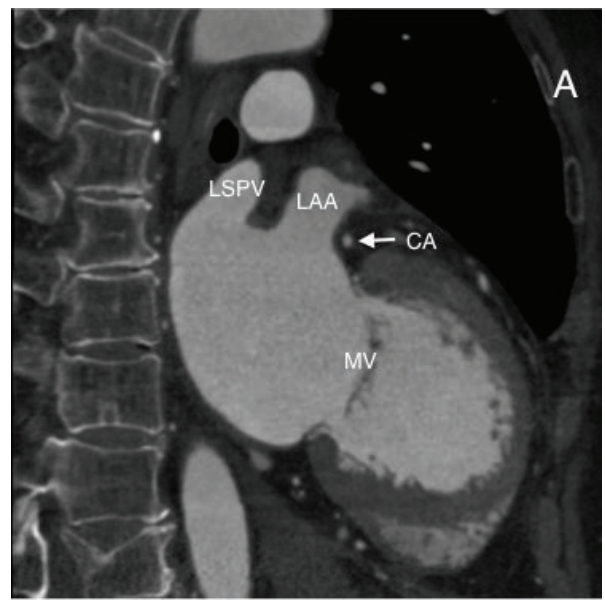

(a)

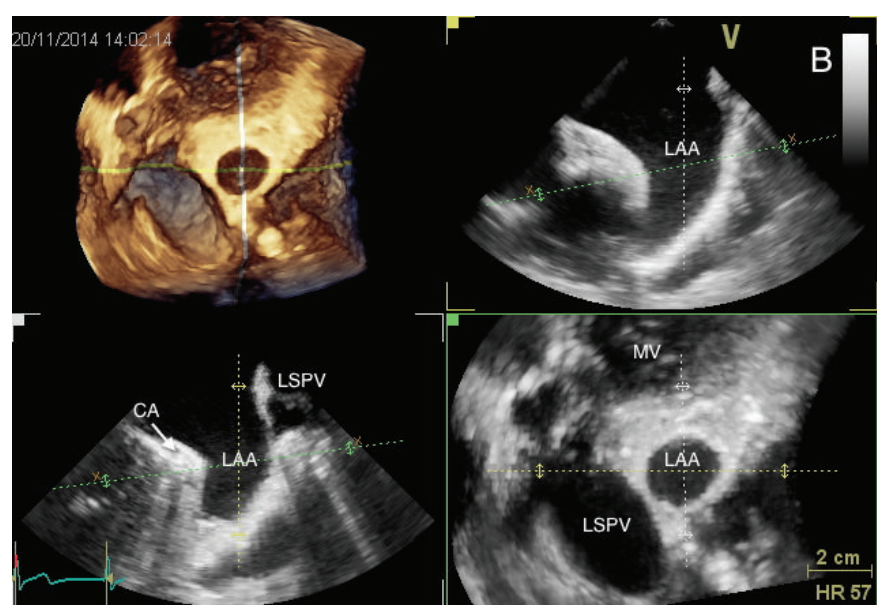

(b)

FIGURE 1: Left appendage anatomical relationship, as seen with cardiac CT scan (a) and 3D transesophageal echocardiography (b). LAA: left atrial appendage; LSPV: left superior pulmonary vein; CA: circumflex artery; MV: mitral valve.

In fact, it is the most common source of cardioembolic stroke in AF as LAA thrombus is present in up to $15 \%$ of patients in $\mathrm{AF}[6]$ and $90 \%$ of thrombus formation in nonvalvular $\mathrm{AF}$ is in LAA [7].

For this reason it has been defined as the "most lethal human appendage" [8] causing significant mortality and morbidity in AF patients.

CHADS2 score (cardiac failure, hypertension, age, diabetes, diabetes, and stroke) and the more recent CHA2DS2VASc score (with the addition of gender, vascular disease) are useful tools to stratify thromboembolic risk in AF patients, guiding the decision for anticoagulation therapy $[9,10]$. In fact, oral anticoagulation (OAC) has been shown to significantly reduce the risk of thromboembolism in numerous studies [11].

However, due to poor patient compliance, contraindications, and potential bleeding complications, OAC is underutilized in AF [12].

So, in certain clinical scenarios, when anticoagulation is contraindicated or has a high risk, LAA percutaneous closure is a safe and effective measure to prevent thromboembolism.

Considering that only $10 \%$ of the clinically relevant emboli in nonvalvular AF do not originate in the LAA [13], with the exclusion of LAA as an embolic source, the remaining small risk does not require any longer OAC with its inherent risk for side effects, especially major bleedings. Numerous methods have been developed to exclude the LAA, surgically or percutaneously, LAA $[14,15]$.

\section{Physiology and Pathophysiology of the LAA}

2.1. Anatomy and Physiology. The LAA is a remnant of the embryonic left atrium [1], lying in the left atrioventricular groove and in close relation with the left circumflex artery, with the left superior pulmonary vein posteriorly, with the mitral valve annulus medially, and with the left phrenic nerve laterally (Figure 1).
Anatomical studies have described numerous shapes of the LAA: as a long, narrow, tubular, and hooked structure [17].

The shape of the LAA ostium is typically elliptical (68.9\%), with a long diameter ranging from 10 to $40 \mathrm{~mm}$ and a maximal depth ranging from 16 to $51 \mathrm{~mm}$ [18]. A round shape is present only in $5.7 \%$ of cases. Interestingly, ostium diameters showed minimal changes during different phases of the cardiac cycle in sinus rhythm (maximal change 1 to $2 \mathrm{~mm}$ ), while no change was observed during AF [19].

Veinot et al. [20] have examined 500 anatomical findings: in more than two-thirds of cases, LAA is composed of two or more lobes, located in different planes. Classically, the lobes head toward the atrioventricular groove and the basal surface of the left ventricle. This has to be kept in mind during imaging studies in order to rule out intracavitary thrombus: failure to view all the lobes or incomplete visualization of a lobe may account for underdiagnosis of LAA thrombosis.

Recently, a CT based study classified LAA morphology on the basis of the presence of a bend, giving to the LAA an appearance similar to a chicken wing ( $48 \%$ of cases). Others possible morphologies are cactus shape (30\%), with a dominant central lobe and secondary lobes extending from the central lobe in both superior and inferior directions; windsock shape (19\%), with 1 dominant lobe; cauliflower shape (3\%), with limited overall length and complex internal characteristics [21].

Histologically, the LAA has a single layer of endothelium and contains pectinate muscles with variable thickness [19]. The anterolateral wall, close to the mitral valve, has the minimum thickness $(0.5 \mathrm{~mm})$ : particular care should be taken to avoid perforation during invasive procedures.

As said before, the LAA does not seem to be just an embryologic remnant, a useless appendage. The LAA is responsible for several functions: it acts as a reservoir during left ventricular systole, a conduit for blood transiting from the pulmonary veins to the left ventricle during early diastole, 


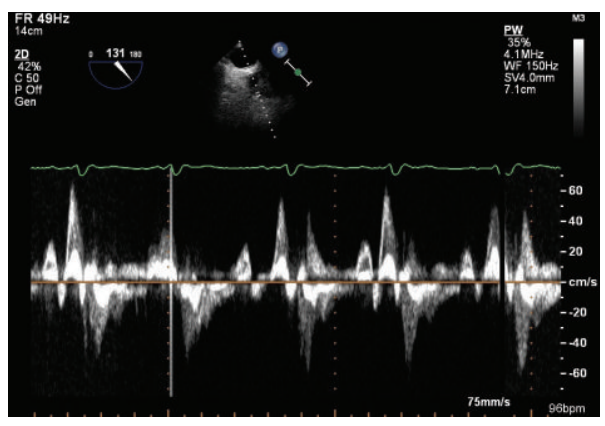

(a)

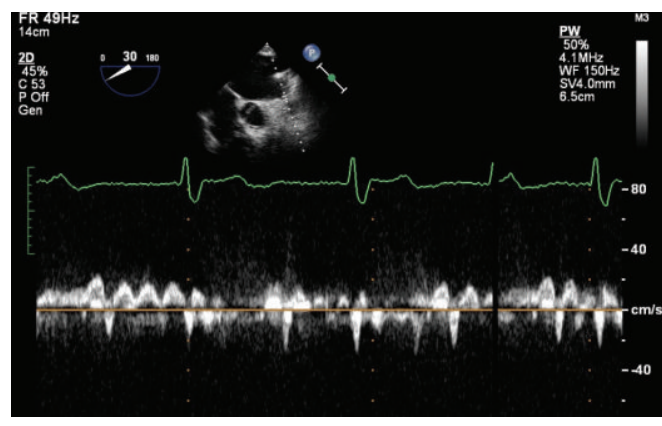

(b)

FIGURE 2: Left atrial appendage emptying velocity during normal sinus rhythm (a) and during atrial fibrillation (b).

an active contractile chamber that augments left ventricular filling in late diastole, and a suction source that refills itself in early systole [22]. In fact, the LAA seems more distensible than the rest of the atrium and it could act as a volume reserve. Experimental findings during cardiac surgery demonstrated how LAA temporary exclusion augments LA pressure [23]. It is also possible that the LAA could contribute to stroke volume, due to its intrinsic contractile capability [24].

The LAA also has an endocrine role: it contains stretchsensitive receptors that are able to influence heart rate and natriuretic peptides secretion in response to change in atrial pressure. A quantitative analysis of atrial natriuretic peptides (ANP) in excised LAAs revealed a content of approximately $30 \%$ of all cardiac ANP [25]. Experimental infusion of fluid in the LAA results in diuresis and natriuresis and increased heart rate, supporting a significant role of the LAA in regulating normal cardiac physiology [26].

However, little is known about these functions in a pathological LAA, as seen during AF or after LAA closure.

The LAA has a distinct pattern of contraction, extensively studied with TEE [27]. It has an augmented contractility in respect to the atrium; typically, it has a biphasic pattern of emptying, a first passive phase in protodiastole and a second active phase during left atrial contraction and a prominent monophasic pattern of filling (Figure 2(a)). During atrial fibrillation, the pattern is characterized by a rapid alternation of emptying and filling, with lower velocities (Figure 2(b)).

Abnormalities of the LAA function observed at TEE in AF (perturbations of LAA emptying peak flow velocity, LAA fractional area change and LAA velocities $<0.2 \mathrm{~m} / \mathrm{sec}$ ), are associated with the occurrence of spontaneous echo-contrast and thrombus formation resulting from blood stagnation in the LA. These findings have been shown to be associated with the occurrence of ischemic strokes in several clinical reports [28].

2.2. Role of the LAA in Cardiac Pathophysiology. As mentioned before, the LAA is the most common source of cardioembolic stroke in nonvalvular AF (up to $90 \%$ of cases) [7].

The reasons why this happens are multiple, summed up by the Virchow triad [29]. First of all, the risk of thrombus formation depends on the hemodynamic function of the LAA. Three LAA flow patterns have been described:

(1) type I, characterized by a regular biphasic emptying pattern, occurring in sinus rhythm;

(2) type II, characterized by a saw-tooth emptying pattern, occurring in some patients with atrial fibrillation;

(3) type III, without any active emptying pattern, typically occurring during AF. This is associated with the highest incidence of spontaneous echo-contrast and thrombus [30].

A reduced LAA peak flow velocity is considered as one of the strongest independent predictors of an increased thromboembolic risk [31].

Furthermore a prothrombotic and hypercoagulable state in $\mathrm{AF}$ has been demonstrated, manifested by increased blood levels of markers, reflecting coagulation activity (e.g., prothrombin fragments 1 and 2, fibrinopeptide A, thrombinantithrombin complexes, and D dimer) [32].

Eventually, atrial fibrillation leads to damages, fibrosis, and inflammation of the endothelium of the left atrium, especially of the LAA [33].

In addition to these factors, LAA shape and size have also been recently evaluated as additive risk factors: in fact spontaneous echo-contrast is most likely found in larger LAA with more complex anatomies [21].

\section{LAA as a Target for Thromboembolic Risk Prevention}

Although antiarrhythmic drugs and catheter ablation are effective in symptomatic relief for patients with atrial fibrillation, the prevention of thromboembolic events is still entrusted to oral anticoagulation (vitamin $\mathrm{K}$ antagonists, VKA), irrespective of the rhythm management strategy.

With the recent emergence of new antithrombotic drugs (i.e., dabigatran, rivaroxaban, apixaban, and edoxaban), it became clear that VKA, although being more effective than aspirin and combination aspirin-clopidogrel, is often not well tolerated by patients, has a very narrow therapeutic range, and has a high risk for bleeding complications. However, 


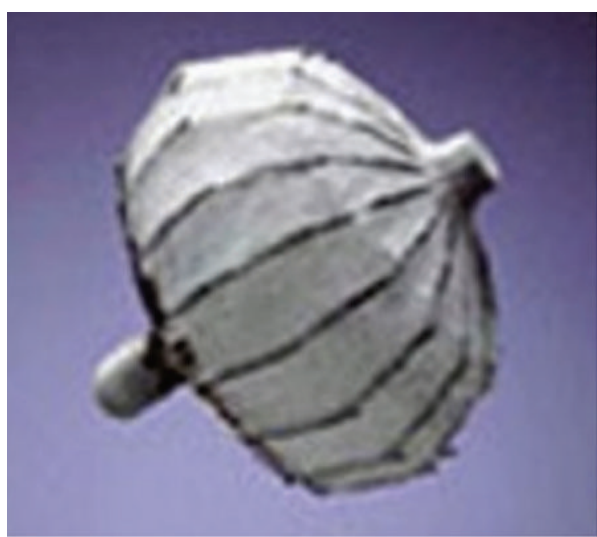

(a)

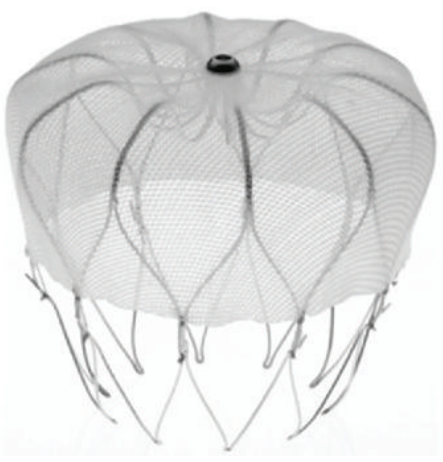

(c)

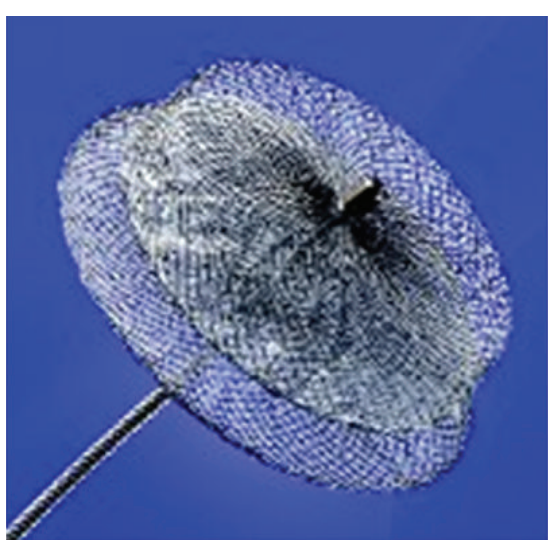

(b)

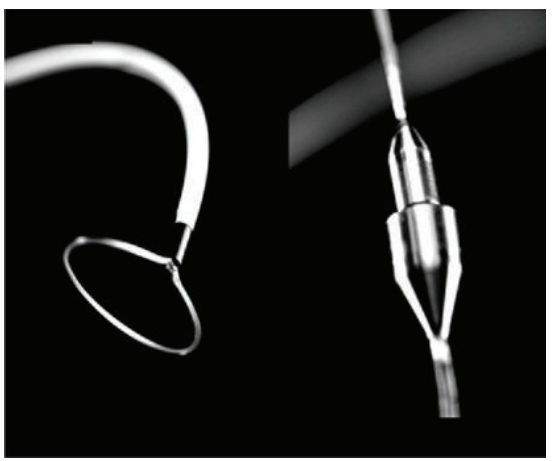

(d)

Figure 3: Left atrial appendage occlusion devices: PLAATO device, no longer available (a), Amplatzer Cardiac Plug (b), Watchman (c), and Lariat device (d).

the drugs mentioned above do not completely solve the risk of bleeding related to antithrombotic therapy.

This is why, over the years, several clinical trials have assessed the feasibility and efficacy of LAA occlusion as a tool for thromboembolism prevention in AF. The first interventions were performed by surgical ligation or removal of the LAA during valvular operations. In fact, LAA obliteration was first suggested as an addition to mitral valvotomy, even before the advent of cardiopulmonary bypass [4]. About fifty years later, interest in surgical LAA exclusion increased after the development of Maze procedure, performed by Cox, that was a reliable solution for the treatment of AF and included atrial appendage removal [34]. Since then, the procedure has evolved in two directions: LAA exclusion with sutures on the epicardial or endocardial surface and LAA excision through staples or removal and oversew. The surgical literature on LAA closure consists primarily of retrospective case series and, regardless of the approach used, showed that incomplete LAA closure may be worse than no closure [35]. All available studies show a failure rate between 10\% [36] and $55 \%$ [37] with the consequent effect of potentially increasing the incidence of stroke.

The vast majority of patients, however, suffers from nonvalvular atrial fibrillation and has no indications for cardiac surgery: this is why in the last decade percutaneous approaches for LAA occlusion were developed. Obstruction of the LAA orifice with an occlusion device [38] or percutaneous suture ligation using an endocardial/epicardial approach [39] is the two alternatives (Figure 3).

The first percutaneous LAA occlusion was performed by the electrophysiologist Michael Lesh with a device called PLAATO (Percutaneous Left Atrial Appendage Transcatheter Occluder) on 30 August 2001 [40].

After that, several studies using the PLAATO device have been published: in the international multicenter feasibility trials [41], device implantation was successful in 108 of 111 patients $(97.3 \%)$ with only one cardiac tamponade and one major vascular complication. The postprocedural stroke incidence was lower than that projected by the clinical scores (mean CHADS2 score 2.5). In fact, the incidence of the major and minor stroke was under $2 \%$ during a mean follow-up period of about one year.

Further studies showed that the PLAATO device appeared to be effective in reducing the stroke risk in patients with $\mathrm{AF}$ (stroke incidence 2.3\%/year versus 6.6\%/year as predicted by CHADS2 score), with a small risk of major periprocedural events (procedural success $90 \%$, cardiac tamponade $3.3 \%$, acute mortality $1.1 \%$, and device embolization $0.6 \%)[42]$.

However, the PLAATO device has been discontinued for commercial reasons and then it was withdrawn from the market. 
The Amplatzer Cardiac Plug (ACP) has the longest clinical follow-up among the currently available LAA occluders [43].

Trials have shown that complications, such as pericardial effusion leading to cardiac tamponade, occurred in $2 \%$ of patients, as did the neurological events (Table 1).

Technical success was $97 \%$ and a relevant thrombus on the device during follow-up TEE was seen in $3 \%$. The percentage of residual peridevice flow is very low: at 6 months, TEE is about $1 \%$, probably due to the device's peculiar shape, with a disk that occludes the so-called "mouth" of the LAA. Generally, antithrombotic therapy after ACPs implant relies on double antiplatelet therapy instead of OAC.

The second generation of Amplatzer device, the so-called Amulet device, has recently been introduced in the market: Amulet data are available in only a few patients, although it was used in over 250 patients in Europe. The device [44, 45] has new features as compared to the first generation ACP as it shows good deliverability ( $96 \%-100 \%$ of procedural success) with $0 \%-5 \%$ pericardial effusion incidence and without acute strokes or device embolization.

The Watchman device is the only one evaluated in prospective, controlled, randomized trials (Table 2) examining its efficacy and safety (PROTECT AF trial [38] and PREVAIL trial [46]).

In the PROTECT AF, that enrolled 707 patients for 1065 patient-years of follow-up, a relative risk reduction of $46 \%$ of ischemic strokes and systemic thromboembolism (from 2.85 to 1.53 ) was observed in comparison to the control group, although a higher rate of adverse safety events was noted, mainly due to periprocedural complications such as pericardial effusion and procedural stroke typically related to air embolism.

Also the PREVAIL study, in patients with higher risk, demonstrated low-early and long-term primary and safety event rates.

Furthermore, a cost-efficacy analysis was carried out and showed that LAA occlusion was cost-effective when compared to warfarin and dabigatran (but only marginally with the latter drug) [47].

A subanalysis of patients enrolled in PROTECT AF showed that residual peridevice flow is possible after device implantation. However, small peridevice residual flow does not seem to have an impact on safety and clinical efficacy of Watchman implantation [48].

The WaveCrest device has recently received a CE mark as well, and it seems to provide a more superficial deployment with little or no manipulation within the LAA body. The WaveCrest device consists of a nitinol structure without exposed metal hub and with a foam layer facing the LAA to promote rapid organization and a PTFE layer facing the LA to reduce thrombus formation. Procedural and follow-up data are not available yet for this device.

The Lariat combined endocardial/epicardial suture ligation of the LAA uses a combination of transseptal placement of a temporary balloon in the LAA, magnet-tipped guidewires inserted into the LAA and the pericardial space, and a closure snare device. This device demonstrated successful LAA closure in a canine model [49]. Studies in the human population [50, 51] in almost 200 patients showed a good procedural success (93-94\%). Early major complications were higher as compared to fully percutaneous devices: the incidence of pericardial effusion requiring pericardiocentesis was between $11 \%$ and $20 \%$, and the incidence of major bleeding was $9 \%$ while another $9 \%$ of patients suffered LAA perforation needing open chest surgery. At the follow-up incidence of stroke, myocardial infarction was under 3\%/year. Furthermore, LAA exclusion with this device appears to reduce AF burden [52], thus confirming the role of the LAA in triggering AF.

\section{Imaging for Left Atrial Appendage Occlusion}

Adequate imaging modalities are essential for successful LAA occlusion, by guiding preprocedural planning and periprocedural assessment and follow-up.

This usually requires TEE or CT. TEE is crucial in guiding the procedure of LAA occlusions [53], and it is recognized as the gold standard for it.

4.1. Preprocedural Assessment. At first, it is important to confirm the absence of thrombi in LAA before the procedure, in order to avoid a possible embolization with sheath or device manipulation.

The imaging technique that is more validated and more often used for this purpose is TEE: in some patients, there may still be difficulties in differentiating prominent pectinate muscles from LAA thrombi. However, the incidence of LAA thrombus (Figure 4(a)) or sludge (Figure 5) among patients undergoing AF ablation who have been adequately anticoagulated was found to be very low, and it is well correlated with the CHA2DS2-VASc score [54].

Dual-enhanced cardiac CT [55] and cardiac MRI [56] could also be useful for this purpose (Figure 4(b)).

Spontaneous echocardiographic contrast is diagnosed in the presence of smoke-like echoes with a characteristic swirling motion, when the gain settings have been increased in a stepwise manner.

A thrombus is diagnosed if an echo reflecting mass is evident in more than one imaging plane, with independent mobility.

If a thrombus is detected inside the LAA, it is prudent to optimize anticoagulation and reassess LAA status after 4 weeks of optimal anticoagulation therapy.

In case of persistence, it is possible to surgically remove the thrombus and exclude the LAA. Percutaneous procedures have also been performed in this setting with an embolic protection device in the supra-aortic trunks [57].

However, it seems prudent not to perform this procedure in case of LAA thrombosis.

Preprocedural TEE guides the decision of the device size: multiplane views (midesophageal $0^{\circ}, 45^{\circ}, 90^{\circ}$, and $135^{\circ}$ ) characterize LAA shape and morphology, facilitated by 3 dimensional reconstructions (Figure 6(a)). 


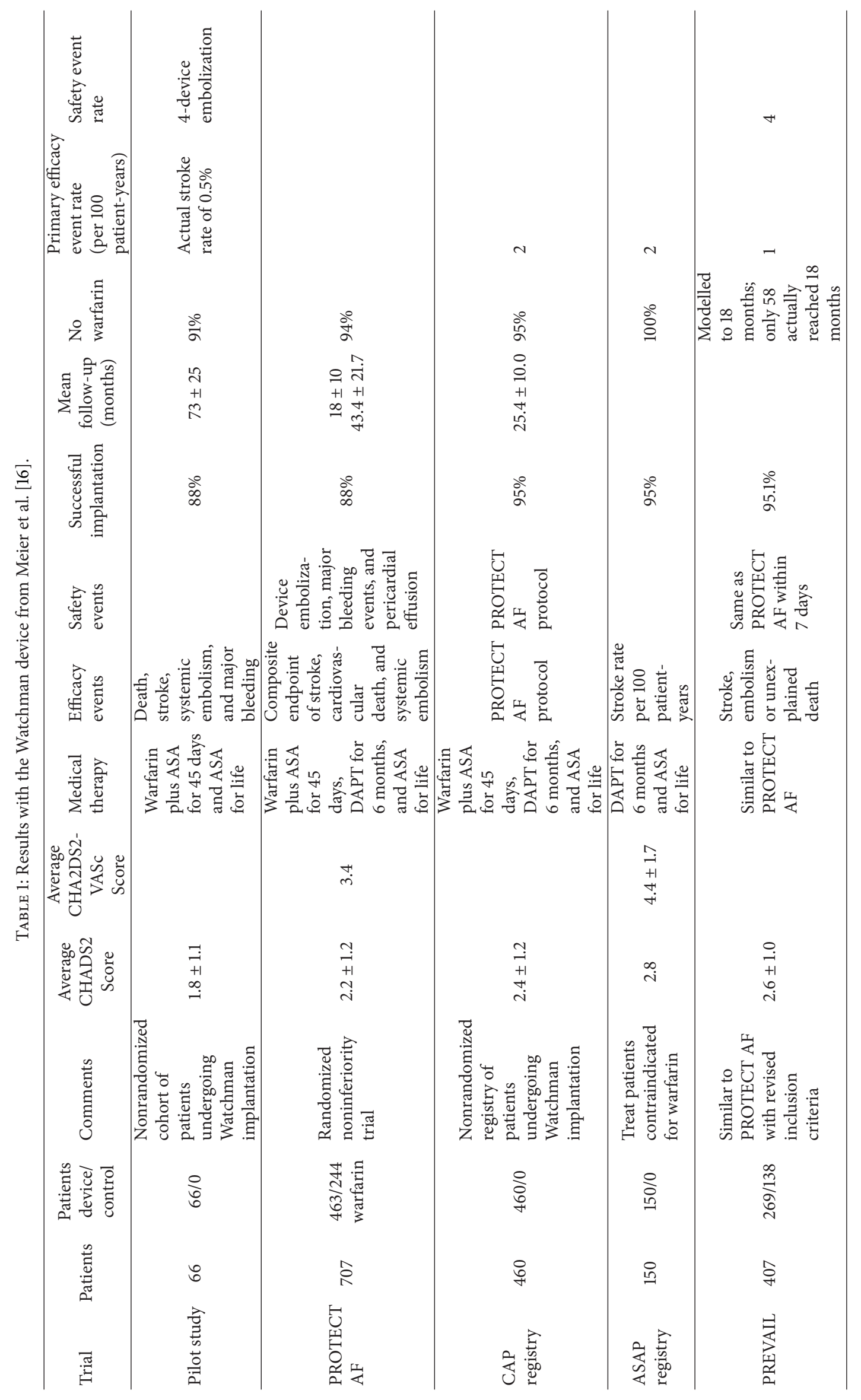




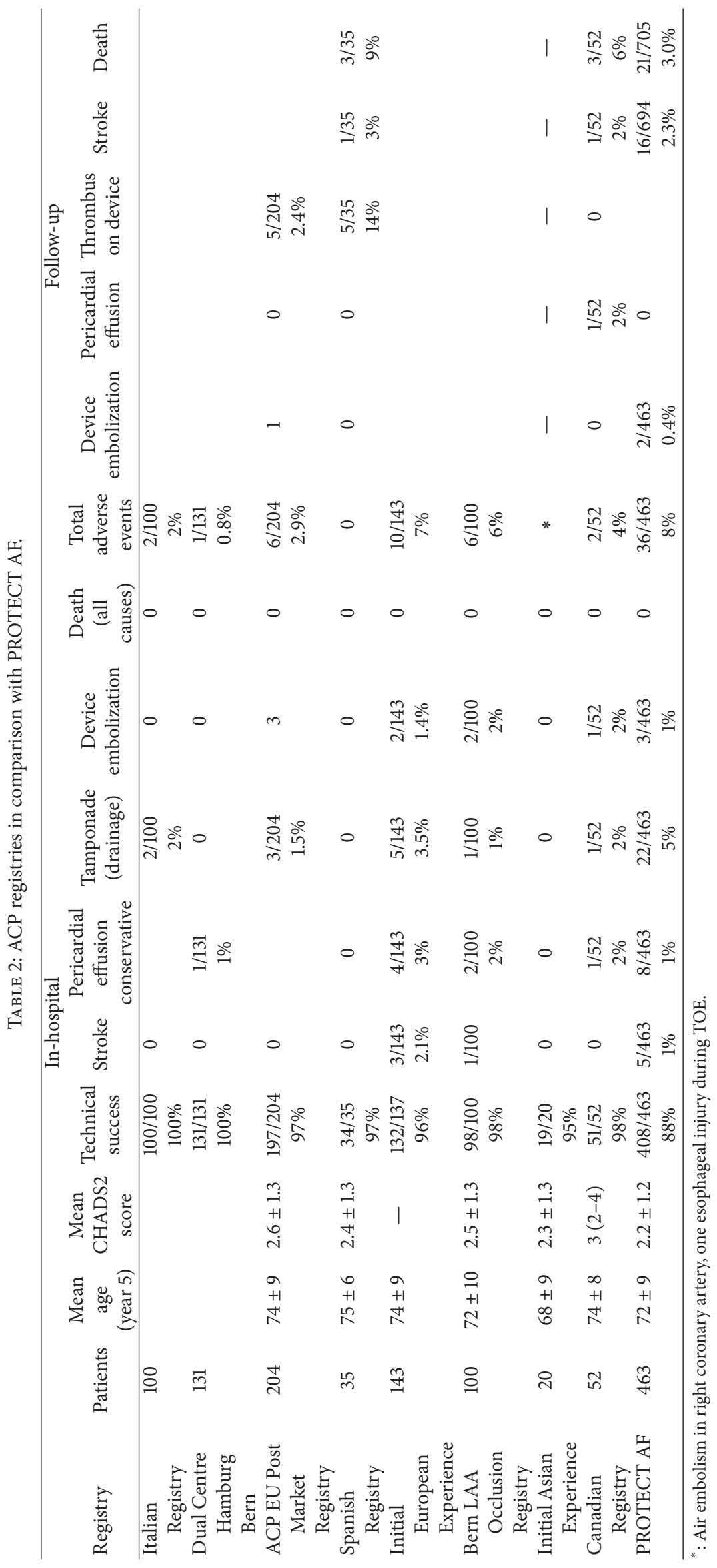




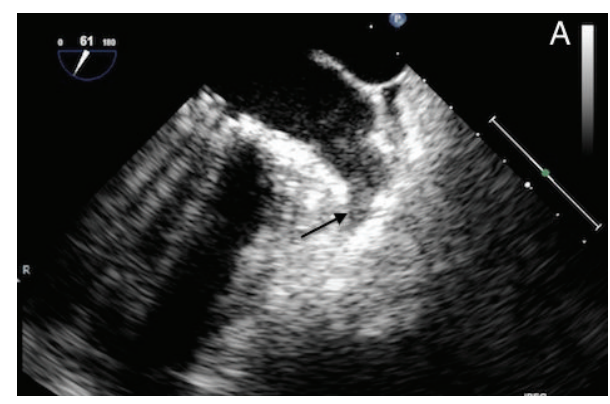

(a)

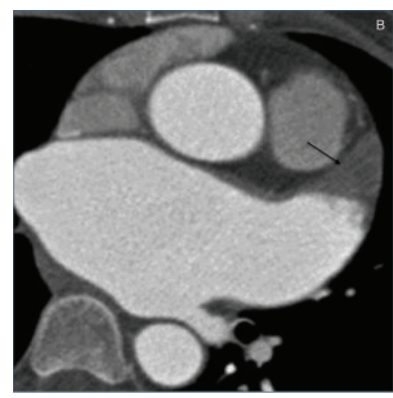

(b)

FIGURE 4: Left atrial appendage thrombosis (arrow), as seen with transesophageal echocardiography (a) and CT scan (b).

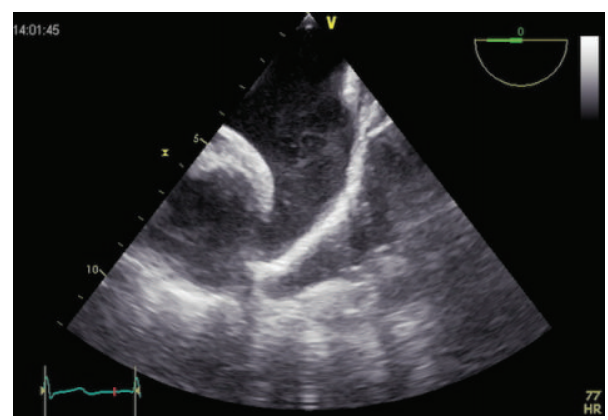

FIGURE 5: Severe spontaneous smoke effect (sludge) in left atrial appendage.

It has to be kept in mind that 2D TEE underestimates true dimensions in comparison to $3 \mathrm{D}$ TEE or CT measurements (Figure 6(b)) [58].

The maximal width of the LAA ostium is measured from the level of the left circumflex coronary artery up to a point at $1-2 \mathrm{~cm}$ from the tip of the left superior pulmonary vein limbus.

The maximal depth is measured from the ostium line to the apex of the LAA. Sizing tables are available for both the Watchman and ACP devices.

The size of the chosen device should at least be $10-20 \%$ larger than the measured diameter: a correct oversizing is essential in order to avoid peridevice flow after deployment; on the other side, excessive oversizing may result in a compression of the left circumflex artery.

It is worth noting that the ostium of the LAA is typically elliptical, while all available occluders have a round shape, possibly accounting for incomplete sealing of the device and possible cause of leakages.

ACP has to be preferred if the depth of the LAA is smaller than the width of the ostium, because the placement of a Watchman device may result in an unstable position.

Preprocedural TEE evaluation could also be useful to better assess the thromboembolic risk of the patient. LAA dimensions, LAA velocities, left atrial dimensions and fibrosis, left ventricular dysfunction, spontaneous echo-contrast, and aortic plaque (especially in aortic arc) have been associated with an increased thromboembolic risk [59]. In particular, the presence of left atrial abnormalities is associated with an embolic risk of 7.8\%/year, as well as a CHA2DS2-VASc score of 5 .

All these data could be useful to guide decisions on thromboembolic risk prevention in case of borderline CHA2DS2-VASc scores.

4.2. Procedural Imaging to Guide Left Atrial Appendage Occlusion. TEE allows us to visualize the LAA during the procedure: it is essential for the positioning and deployment of the device.

The majority of centers perform the procedure under general anesthesia with TEE and fluoroscopic guidance. There are only few reports of intracardiac echocardiographic guidance during percutaneous LAA occlusion.

TEE facilitates the transseptal puncture (Figure 7) and especially 3D TEE can provide a real-time full view of the LAA, the shape of the ostium (Figure 8), with accurate measurements of the landing zone.

A final decision on device size is based on the information collected with all imaging modalities: echocardiography, fluoroscopy, and CT.

After deployment, a tug test should be performed demonstrating simultaneous movement of the device and appendage (Figure 9). Optimally, the device should not protrude $>4-7 \mathrm{~mm}$ beyond the LAA ostium, and residual flow should be $<5 \mathrm{~mm}$ by color Doppler with a compression grade of $8-$ $20 \%$, expressed in percent comparing the diameter of the implanted device with the unconstricted diameter indicated by the manufacturer. When optimal positioning is confirmed, the device is released. Rare device embolization after mobilization of the patient has been observed.

Following successful device deployment, the pericardium is evaluated for effusion.

4.3. Follow-Up Imaging. Postprocedural imaging aims to assess device position, peridevice residual flow in the LAA, and thrombus formation on the device.

TEE (Figure 10) and CT can both be used for this purpose.

In the PROTECT AF trial, serial TEE imaging was performed at 45 days, 6 months, and 1 year following implant [38].

Residual peridevice flow is common ( $41 \%$ at 45 days) in patients treated with the Watchman device. It is unclear if this 


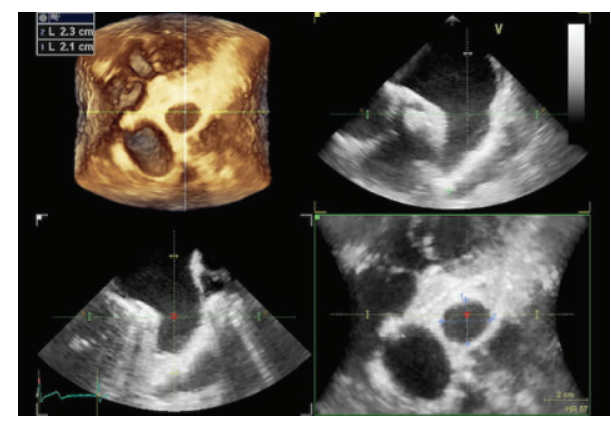

(a)

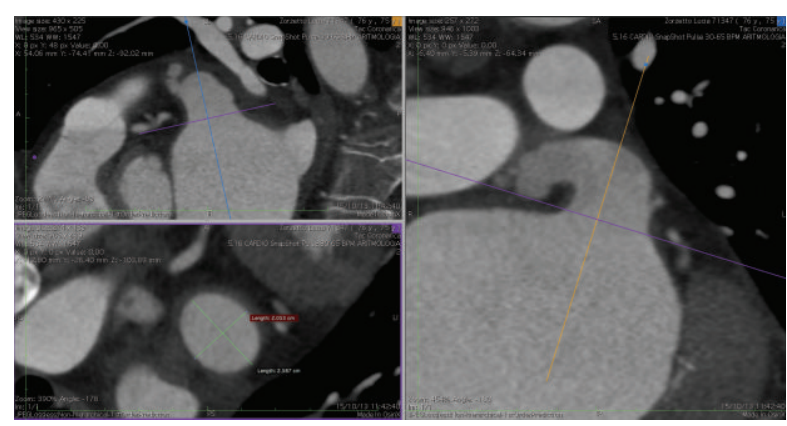

(b)

FIGURE 6: Left atrial appendage measures with transesophageal echocardiography (a) and CT scan (b).

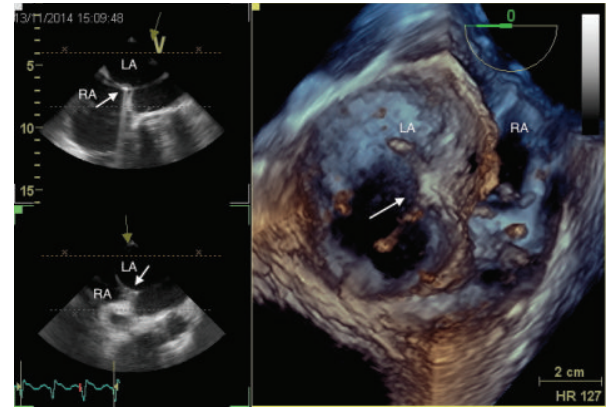

FIGURE 7: Real-time 3D echocardiography during transseptal puncture. The tip of the catheter (arrow) is passing from the right atrium (RA) to the left atrium (LA), through interatrial septum.

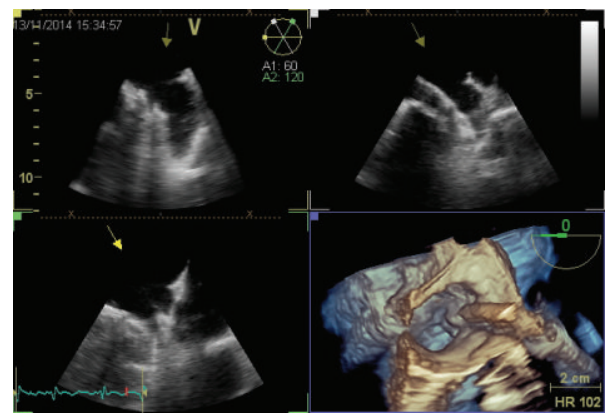

FIgURE 8: Progress of the delivery system in the left atrial appendage.

could be related to possible thromboembolic events, since new thrombi may be formed in the distal LAA pouch [48]. Of note in the PROTECT AF trial, patients with a peridevice flow did not have a worse clinical outcome, regardless of the chosen antithrombotic therapy (warfarin, double antiplatelet agents, ASA).

With Amplatzer devices, as the disc of the device typically covers the entire LAA ostium (pacifier principle), residual peridevice leaks are less frequent.

4.4. Anticoagulation after Implantation. Postprocedural antithrombotic therapy with warfarin or dual antiplatelet drugs

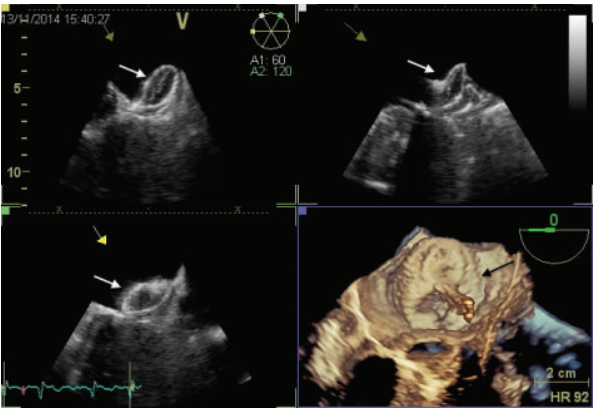

Figure 9: The image shows the so-called "tug test." An Amplatzer Cardiac Plug is pulled before the deployment. During this maneuver, the distal part of the device ("disk," arrow) is put in tension, while the distal part ("lobe") remains anchored in left atrial appendage.

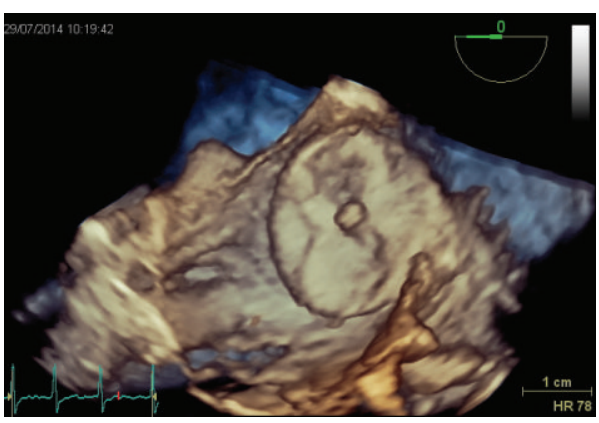

Figure 10: An Amplatzer Cardiac Plug six months after implant, with perfect sealing and endothelization.

is recommended after implantation to avoid thrombus formation on the device until completion of endothelialization, provided there are no contraindications. For the Watchman device, the antithrombotic protocol of the PROTECT AF trial is adopted: 45 days after implantation warfarin was discontinued and substituted by ASA + clopidogrel if the TEE showed the absence of thrombi or a residual peridevice flow of $<5 \mathrm{~mm}$ in width; clopidogrel was stopped if the 6 months TEE follow-up demonstrated no complications.

Usually, one antiplatelet agent is continued indefinitely, as most patients are elderly with evidence of atherosclerotic disease, although the bleeding risk must be considered. 
The PREVAIL trial and ASAP study provided evidences for double antiplatelet therapy instead of VKA for the Watchman device.

Observational studies with the ACP followed a regimen of clopidogrel + ASA for 1 month and acetylsalicylic acid for 3 to 6 months [60], borrowing the antithrombotic protocol from the experience with the Amplatzer PFO Occluder. In patients who are treated with antiplatelets drugs, it is reasonable to perform an imaging test (TEE or CT scan) before clopidogrel termination and again if ASA cessation is planned.

Incomplete LAA occlusion could create, theoretically, a thrombus-containing pocket with a source of possible systemic embolization. Anyway, as mentioned above, small residual shunts $(<5 \mathrm{~mm})$ are usually considered irrelevant and may close spontaneously with time. When all patients with residual shunts are included, the stroke risk is no different compared with patients in whom the LAA is completely occluded regardless of antithrombotic therapy [48]. However, this remains a field open for debate.

\section{Indications for Left Atrial Appendage Occlusion}

PROTECT AF and PREVAIL randomized controlled trials were included in the recent ESC focused guidelines on stroke prevention in patients with atrial fibrillation. In fact, they suggest to use the CHA2DS2-VASc risk score $>1$ as the threshold value for considering LAA occlusion [16].

Individual risk-benefit evaluation is fundamental, bearing in mind that the use of OAC has a primary recommendation.

5.1. When Anticoagulation Is Not Possible. Patients with a high thromboembolic risk (CHA2DS2-VASc score of $\geq 2$ ) but contraindication to systemic anticoagulation (e.g., history of intracranial or life threatening bleeding, coagulation disorders) represent the most accepted indication for LAA occlusion. In a survey of European centers, the most common indication for LAA closure was an absolute contraindication to OAC [61].

So far, no randomized trials targeting this specific group of patients are available; in fact, this is the result of several observational studies and registries. However, the significant bleeding risk of dual antiplatelet therapy, indicated for 1 or 6 months after implantation, has to be considered [62]. Generally, this is only for a short time, thus reducing the cumulative risk of major bleeding events.

In patients who cannot receive any antiplatelet agent, the Lariat technique can be considered.

5.2. When Oral Anticoagulation Is Possible. This is the only indication, as cited above, that is based on randomized controlled trials. Those patients, in whom OAC or NOAC are considered to pose an unacceptable bleeding risk (HASBLED $\geq 3$ ), but with high stroke risk (CHA2DS2-VASc score of $\geq 2$ ), should be considered for LAA occlusion.
The possibility of LAA occlusion should be discussed with the patient, remembering that anticoagulation currently remains the standard of therapy.

Patients should be elucidated about the possibility of therapy with NOAC, that, compared to OAC, have at least an equivalent and probably improved efficacy, with lower rate of intracranial and, for some, lower overall bleeding risk.

It should be emphasized that we do not have any direct data comparing NOAC with LAA occlusion.

Ultimately, the decision belongs to a well-informed patient in collaboration with the physician.

The HAS-BLED score does not characterize the bleeding risk in certain categories of patients (e.g., patients with cancer or chronic inflammatory bowel disease): to these, LAA occlusion may also be offered.

Another possible scenario in which LAA occlusion could be helpful is the setting of triple anticoagulant therapy due to coronary stent interventions in AF patients as it poses a significant rise in bleeding risk [63].

End-stage renal failure poses patients at a high stroke risk and high bleeding risk: LAA occlusion could be a debatable alternative, keeping in mind that all NOAC are contraindicated with creatinine clearance $<15 \mathrm{~mL} / \mathrm{min}$ and warfarin could increase tissue calcification and enhanced atherosclerosis in this setting.

5.3. As a Complement to Anticoagulation. The combination of LAA occlusion and OAC is debated and occasionally performed in patients with embolic events despite the adequate antithrombotic therapy, provided that there are no other plausible causes (e.g., patients with mechanical prosthetic valves with evidence of thrombus in the LAA).

\section{Conclusions}

The LAA is considered the "most lethal human appendage" as it causes significant mortality and morbidity in AF patients. We have to learn more about its complex role in physiology and pathology.

However, LAA occlusion is becoming an interesting tool in reducing thromboembolic risk in certain categories of patients, as it has become a safe and effective procedure.

Indeed, we need new randomized prospective trials comparing LAA procedure with NOAC, as they have a safety profile better than old OAC.

Other interesting aspects that warrant investigation are the clinical significance of residual peridevice flow and the correct antithrombotic therapy after LAA closure.

In this way, we can shed light on thromboembolism management in AF, in order to improve our knowledge when choosing between different measures to reduce the risk of catastrophic strokes.

\section{Conflict of Interests}

The authors declare that there is no conflict of interests regarding the publication of this paper. 


\section{Acknowledgement}

The authors want to sincerely thank St. Jude Medical Italia Spa for the scientific and economic support supplied to the writing of this paper.

\section{References}

[1] H. M. F. Sherif, "The developing pulmonary veins and left atrium: implications for ablation strategy for atrial fibrillation," European Journal of Cardio-Thoracic Surgery, vol. 44, no. 5, Article ID ezt098, pp. 792-799, 2013.

[2] S. Qamruddin, J. Shinbane, J. Shriki, and T. Z. Naqvi, "Left atrial appendage: structure, function, imaging modalities and therapeutic options," Expert Review of Cardiovascular Therapy, vol. 8, no. 1, pp. 65-75, 2010.

[3] B. D. Hoit, Y. Shao, and M. Gabel, "Influence of acutely altered loading conditions on left atrial appendage flow velocities," Journal of the American College of Cardiology, vol. 24, no. 4, pp. 1117-1123, 1994.

[4] J. L. Madden, "Resection of the left auricular appendix; a prophylaxis for recurrent," The Journal of the American Medical Association, vol. 140, no. 9, pp. 769-772, 1949.

[5] Y. Takahashi, P. Sanders, M. Rotter, and M. Haïssaguerre, "Disconnection of the left atrial appendage for elimination of foci maintaining atrial fibrillation," Journal of Cardiovascular Electrophysiology, vol. 16, no. 8, pp. 917-919, 2005.

[6] R. Mahajan, A. G. Brooks, T. Sullivan et al., "Importance of the underlying substrate in determining thrombus location in atrial fibrillation: implications for left atrial appendage closure," Heart, vol. 98, no. 15, pp. 1120-1126, 2012.

[7] J. L. Blackshear and J. A. Odell, "Appendage obliteration to reduce stroke in cardiac surgical patients with atrial fibrillation," Annals of Thoracic Surgery, vol. 61, no. 2, pp. 755-759, 1996.

[8] W. D. Johnson, A. K. Ganjoo, C. D. Stone, R. C. Srivyas, and M. Howard, "The left atrial appendage: our most lethal human attachment! Surgical implications," European Journal of Cardiothoracic Surgery, vol. 17, no. 6, pp. 718-722, 2000.

[9] B. F. Gage, A. D. Waterman, W. Shannon, M. Boechler, M. W. Rich, and M. J. Radford, "Validation of clinical classification schemes for predicting stroke: results from the National Registry of Atrial Fibrillation," The Journal of the American Medical Association, vol. 285, no. 22, pp. 2864-2870, 2001.

[10] G. Y. H. Lip, R. Nieuwlaat, R. Pisters, D. A. Lane, and H. J. G. M. Crijns, "Refining clinical risk stratification for predicting stroke and thromboembolism in atrial fibrillation using a novel risk factor-based approach: the Euro Heart Survey on atrial fibrillation," Chest, vol. 137, no. 2, pp. 263-272, 2010.

[11] R. G. Hart, O. Benavente, R. McBride, and L. A. Pearce, "Antithrombotic therapy to prevent stroke in patients with atrial fibrillation: a meta-analysis," Annals of Internal Medicine, vol. 131, no. 7, pp. 492-501, 1999.

[12] T. J. Bungard, W. A. Ghali, K. K. Teo, F. A. McAlister, and R. T. Tsuyuki, "Why do patients with atrial fibrillation not receive warfarin?" Archives of Internal Medicine, vol. 160, no. 1, pp. 4146, 2000.

[13] L. M. Tsai, J. H. Chen, L. J. Lin, and Y. J. Yang, "Role of transesophageal echocardiography in detecting left atrial thrombus and spontaneous echo contrast in patients with mitral valve disease or non-rheumatic atrial fibrillation," Journal of the Formosan Medical Association, vol. 89, no. 4, pp. 270-274, 1990.
[14] T. Lewalter, R. Ibrahim, B. Albers, and A. J. Camm, "An update and current expert opinions on percutaneous left atrial appendage occlusion for stroke prevention in atrial fibrillation," Europace, vol. 15, no. 5, pp. 652-656, 2013.

[15] S. Chatterjee, J. C. Alexander, P. J. Pearson, and T. Feldman, "Left atrial appendage occlusion: lessons learned from surgical and transcatheter experiences," Annals of Thoracic Surgery, vol. 92, no. 6, pp. 2283-2292, 2011.

[16] B. Meier, Y. Blaauw, A. A. Khattab et al., "EHRA/EAPCI expert consensus statement on catheter-based left atrial appendage occlusion," Europace, vol. 16, no. 10, pp. 1397-1416, 2014.

[17] G. Ernst, C. Stöllberger, F. Abzieher et al., "Morphology of the left atrial appendage," Anatomical Record, vol. 242, no. 4, pp. 553-561, 1995.

[18] P. Su, K. P. McCarthy, and S. Y. Ho, "Occluding the left atrial appendage: anatomical considerations," Heart, vol. 94, no. 9, pp. 1166-1170, 2008.

[19] P. Santangeli, L. Di Biase, R. Horton, J. D. Burkhardt, and A. Natale, "CT imaging to assess the left atrial appendage anatomy: clinical implications," in Computed TomographyClinical Applications, L. Saba, Ed., InTech Open Access Publishing, Vienna, Austria, 2012.

[20] J. P. Veinot, P. J. Harrity, F. Gentile et al., "Anatomy of the normal left atrial appendage: a quantitative study of age-related changes in 500 autopsy hearts: implications for echocardiographic examination," Circulation, vol. 96, no. 9, pp. 3112-3115, 1997.

[21] L. di Biase, P. Santangeli, M. Anselmino et al., "Does the left atrial appendage morphology correlate with the risk of stroke in patients with atrial fibrillation? Results from a Multicenter Study," Journal of the American College of Cardiology, vol. 60, no. 6, pp. 531-538, 2012.

[22] P. Barbier, S. B. Solomon, N. B. Schiller, and S. A. Glantz, "Left atrial relaxation and left ventricular systolic function determine left atrial reservoir function," Circulation, vol. 100, no. 4, pp. 427-436, 1999.

[23] T. Tabata, T. Oki, H. Yamada et al., "Role of left atrial appendage in left atrial reservoir function as evaluated by left atrial appendage clamping during cardiac surgery," American Journal of Cardiology, vol. 81, no. 3, pp. 327-332, 1998.

[24] P. Massoudy, S. Beblo, P. Raschke, S. Zahler, and B. F. Becker, "Influence of intact left atrial appendage on hemodynamic parameters of isolated guinea pig heart," European Journal of Medical Research, vol. 3, no. 10, pp. 470-474, 1998.

[25] C. Chapeau, J. Gutkowska, P. W. Schiller et al., "Localization of immunoreactive synthetic atrial natriuretic factor (ANF) in the heart of various animal species," Journal of Histochemistry and Cytochemistry, vol. 33, no. 6, pp. 541-550, 1985.

[26] C. T. Kappagoda, R. J. Linden, and H. M. Snow, "The effect of distending the atrial appendages on urine flow in the dog," Journal of Physiology, vol. 227, no. 1, pp. 233-242, 1972.

[27] C. Pollick and D. Taylor, "Assessment of left atrial appendage function by transesophageal echocardiography: implications for the development of thrombus," Circulation, vol. 84, no. 1, pp. 223-231, 1991.

[28] M. Zabalgoitia, J. L. Halperin, L. A. Pearce, J. L. Blackshear, R. W. Asinger, and R. G. Hart, "Transesophageal echocardiographic correlates of clinical risk of thromboembolism in nonvalvular atrial fibrillation," Journal of the American College of Cardiology, vol. 31, no. 7, pp. 1622-1626, 1998.

[29] T. Watson, E. Shantsila, and G. Y. Lip, "Mechanisms of thrombogenesis in atrial fibrillation: Virchow's triad revisited," The Lancet, vol. 373, no. 9658, pp. 155-166, 2009. 
[30] M. A. Garcia-Fernandez, E. G. Torrecilla, D. S. Roman et al., "Left atrial appendage Doppler flow patterns: implications on thrombus formation," American Heart Journal, vol. 124, no. 4, pp. 955-961, 1992.

[31] T. Takada, M. Yasaka, K. Nagatsuka, K. Minematsu, and T. Yamaguchi, "Blood flow in the left atrial appendage and embolic stroke in nonvalvular atrial fibrillation," European Neurology, vol. 46, no. 3, pp. 148-152, 2001.

[32] H. Inoue, T. Nozawa, K. Okumura, L. Jong-Dae, A. Shimizu, and K. Yano, "Prothrombotic activity is increased in patients with nonvalvular atrial fibrillation and risk factors for embolism," Chest, vol. 126, no. 3, pp. 687-692, 2004.

[33] A. Frustaci, C. Chimenti, F. Bellocci, E. Morgante, M. A. Russo, and A. Maseri, "Histological substrate of atrial biopsies in patients with lone atrial fibrillation," Circulation, vol. 96, no. 4, pp. 1180-1184, 1997.

[34] J. L. Cox, R. B. Schuessler, H. J. D’Agostino Jr. et al., “The surgical treatment of atrial fibrillation: III. Development of a definitive surgical procedure," Journal of Thoracic and Cardiovascular Surgery, vol. 101, no. 4, pp. 569-583, 1991.

[35] A. G. Dawson, S. Asopa, and J. Dunning, "Should patients undergoing cardiac surgery with atrial fibrillation have left atrial appendage exclusion?" Interactive Cardiovascular and Thoracic Surgery, vol. 10, no. 2, pp. 306-311, 2010.

[36] M. A. García-Fernández, E. Pérez-David, J. Quiles et al., "Role of left atrial appendage obliteration in stroke reduction in patients with mitral valve prosthesis: a transesophageal echocardiographic study," Journal of the American College of Cardiology, vol. 42, no. 7, pp. 1253-1258, 2003.

[37] J. S. Healey, E. Crystal, A. Lamy et al., "Left Atrial Appendage Occlusion Study (LAAOS): results of a randomized controlled pilot study of left atrial appendage occlusion during coronary bypass surgery in patients at risk for stroke," The American Heart Journal, vol. 150, no. 2, pp. 288-293, 2005.

[38] D. R. Holmes, V. Y. Reddy, Z. G. Turi et al., "Percutaneous closure of the left atrial appendage versus warfarin therapy for prevention of stroke in patients with atrial fibrillation: a randomised non-inferiority trial," The Lancet, vol. 374, no. 9689, pp. 534-542, 2009.

[39] K. Bartus, J. Bednarek, J. Myc et al., "Feasibility of closed-chest ligation of the left atrial appendage in humans," Heart Rhythm, vol. 8, no. 2, pp. 188-193, 2011.

[40] H. Sievert, M. D. Lesh, T. Trepels et al., "Percutaneous left atrial appendage transcatheter occlusion to prevent stroke in highrisk patients with atrial fibrillation: early clinical experience," Circulation, vol. 105, no. 16, pp. 1887-1889, 2002.

[41] S. H. Ostermayer, M. Reisman, P. H. Kramer et al., "Percutaneous Left Atrial Appendage Transcatheter Occlusion (PLAATO System) to prevent stroke in high-risk patients with non-rheumatic atrial fibrillation: results from the international multi-center feasibility trials," Journal of the American College of Cardiology, vol. 46, no. 1, pp. 9-14, 2005.

[42] Y. Bayard, H. Omran, P. Neuzil et al., "PLAATO (Percutaneous Left Atrial Appendage Transcatheter Occlusion) for prevention of cardioembolic stroke in non-anticoagulation eligible atrial fibrillation patients: results from the European PLAATO study," EuroIntervention, vol. 6, no. 2, pp. 220-226, 2010.

[43] F. Nietlispach, S. Gloekler, R. Krause et al., "Amplatzer left atrial appendage occlusion: single center 10-year experience," Catheterization and Cardiovascular Interventions, vol. 82, no. 2, pp. 283-289, 2013.
[44] S. C. Lam, S. Bertog, S. Gafoor et al., "Left atrial appendage closure using the amulet device: an initial experience with the second generation amplatzer cardiac plug," Catheterization and Cardiovascular Interventions, vol. 85, no. 2, pp. 297-303, 2015.

[45] X. Freixa, A. Abualsaud, J. Chan et al., "Left atrial appendage occlusion: initial experience with the Amplatzer Amulet," International Journal of Cardiology, vol. 174, no. 3, pp. 492-496, 2014.

[46] D. R. Holmes, S. Kar, M. J. Price et al., "Prospective randomized evaluation of the watchman left atrial appendage closure device in patients with atrial fibrillation versus long-term warfarin therapy," Journal of the American College of Cardiology, vol. 64, no. 1, pp. 1-12, 2013.

[47] S. M. Singh, A. Micieli, and H. C. Wijeysundera, "Economic evaluation of percutaneous left atrial appendage occlusion, dabigatran, and warfarin for stroke prevention in patients with nonvalvular atrial fibrillation," Circulation, vol. 127, no. 24, pp. 2414-2423, 2013.

[48] J. F. Viles-Gonzalez, S. Kar, P. Douglas et al., "The clinical impact of incomplete left atrial appendage closure with the watchman device in patients with atrial fibrillation: a PROTECT AF (percutaneous closure of the left atrial appendage versus warfarin therapy for prevention of stroke in patients with atrial fibrillation) substudy," Journal of the American College of Cardiology, vol. 59, no. 10, pp. 923-929, 2012.

[49] R. J. Lee, K. Bartus, and S. J. Yakubov, "Catheter-based left atrial appendage (LAA) ligation for the prevention of embolic events arising from the LAA initial experience in a canine model," Circulation: Cardiovascular Interventions, vol. 3, no. 3, pp. 224229, 2010.

[50] M. A. Miller, S. R. Gangireddy, S. K. Doshi et al., "Multicenter study on acute and long-term safety and efficacy of percutaneous left atrial appendage closure using an epicardial suture snaring device," Heart Rhythm, vol. 11, no. 11, pp. 1853-1859, 2014.

[51] M. J. Price, D. N. Gibson, S. J. Yakubov et al., "Early safety and efficacy of percutaneous left atrial appendage suture ligation: results from the U.S. transcatheter LAA ligation consortium," Journal of the American College of Cardiology, vol. 64, no. 6, pp. 565-572, 2014.

[52] M. R. Afzal, A. Kanmanthareddy, M. Earnest et al., "Impact of left atrial appendage exclusion using an epicardial ligation system (LARIAT) on atrial fibrillation burden in patients with cardiac implantable electronic devices," Heart Rhythm, vol. 12, no. 1, pp. 52-59, 2015.

[53] B. Meier, I. Palacios, S. Windecker et al., "Transcatheter left atrial appendage occlusion with Amplatzer devices to obviate anticoagulation in patients with atrial fibrillation," Catheterization and Cardiovascular Interventions, vol. 60, no. 3, pp. 417-422, 2003.

[54] S. Puwanant, B. C. Varr, K. Shrestha et al., "Role of the CHADS2 score in the evaluation of thromboembolic risk in patients with atrial fibrillation undergoing transesophageal echocardiography before pulmonary vein isolation," Journal of the American College of Cardiology, vol. 54, no. 22, pp. 2032-2039, 2009.

[55] J. Hur, Y. J. Kim, H.-J. Lee et al., “Cardioembolic stroke: dualenergy cardiac CT for differentiation of left atrial appendage thrombus and circulatory stasis," Radiology, vol. 263, no. 3, pp. 688-695, 2012.

[56] H. Ohyama, N. Hosomi, T. Takahashi et al., "Comparison of magnetic resonance imaging and transesophageal echocardiography in detection of thrombus in the left atrial appendage," Stroke, vol. 34, no. 10, pp. 2436-2439, 2003. 
[57] S. S. I. Bokhari, P. Martinez-Clark, J. P. Zambrano, M. Tracy, and W. W. O'Neill, "Percutaneous mechanical thrombectomy of left atrial appendage thrombus with bilateral neuro-embolic protection followed by closure of left atrial appendage," EuroIntervention, vol. 8, no. 3, pp. 408-409, 2012.

[58] G. Nucifora, F. F. Faletra, F. Regoli et al., "Evaluation of the left atrial appendage with real-time 3-dimensional transesophageal echocardiography: implications for catheter-based left atrial appendage closure," Circulation: Cardiovascular Imaging, vol. 4, no. 5, pp. 514-523, 2011.

[59] The Stroke Prevention in Atrial Fibrillation Investigators Committee on Echocardiography, "Transesophageal echocardiographic correlates of thromboembolism in high- risk patients with nonvalvular atrial fibrillation," Annals of Internal Medicine, vol. 128, no. 8, pp. 639-687, 1998.

[60] E. E. Guérios, M. Schmid, S. Gloekler et al., "Left atrial appendage closure with the Amplatzer cardiac plug in patients with atrial fibrillation," Arquivos Brasileiros de Cardiologia, vol. 98, no. 6, pp. 528-536, 2012.

[61] G. Y. H. Lip, N. Dagres, A. Proclemer, J. H. Svendsen, L. Pison, and C. Blomstrom-Lundqvist, "Left atrial appendage occlusion for stroke prevention in atrial fibrillation in Europe: results of the European Heart Rhythm Association survey," Europace, vol. 15, no. 1, pp. 141-143, 2013.

[62] J. S. Healey, R. G. Hart, J. Pogue et al., "Risks and benefits of oral anticoagulation compared with clopidogrel plus aspirin in patients with atrial fibrillation according to stroke risk: the atrial fibrillation clopidogrel trial with irbesartan for prevention of vascular events (ACTIVE-W)," Stroke, vol. 39, no. 5, pp. 14821486, 2008.

[63] K. Huber, K. Juhani Airaksinen, T. Cuisset, F. Marín, A. Rubboli, and G. Y. H. Lip, "Antithrombotic therapy in patients with atrial fibrillation undergoing coronary stenting: similarities and dissimilarities between north America and Europe," Thrombosis and Haemostasis, vol. 106, no. 4, pp. 569-571, 2011. 


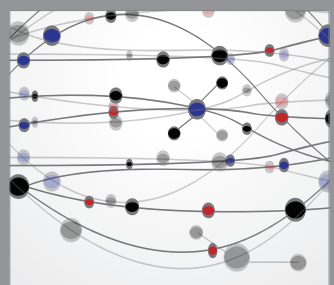

The Scientific World Journal
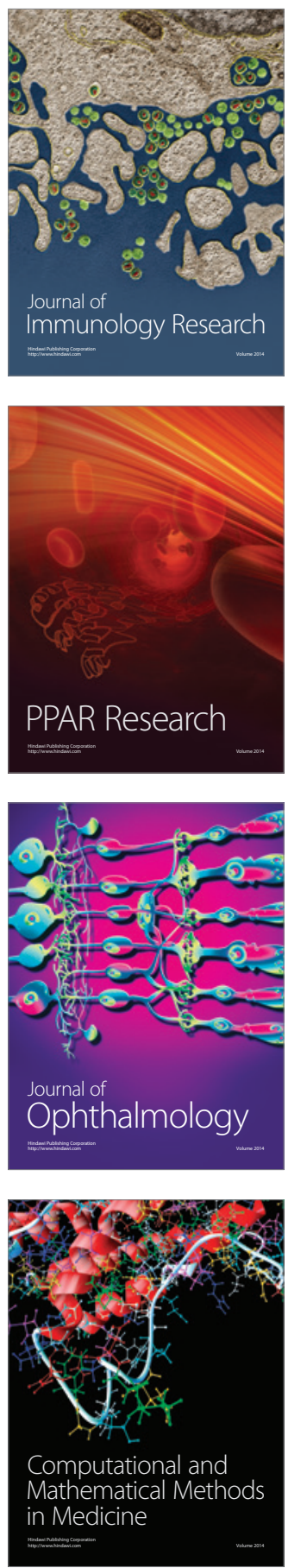

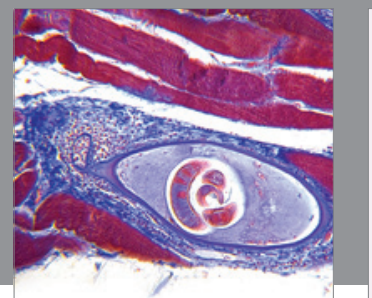

Gastroenterology

Research and Practice
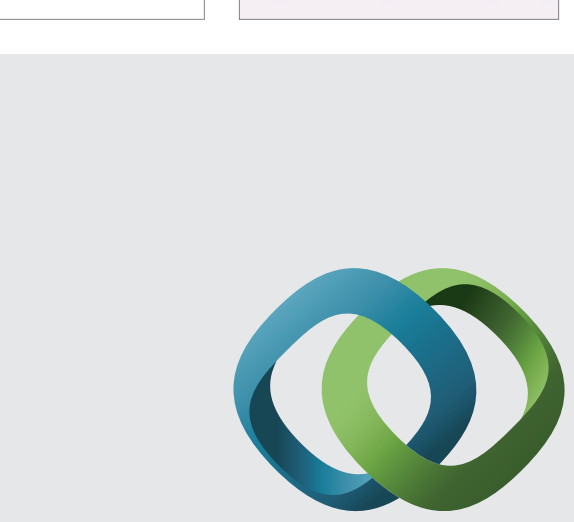

\section{Hindawi}

Submit your manuscripts at

http://www.hindawi.com
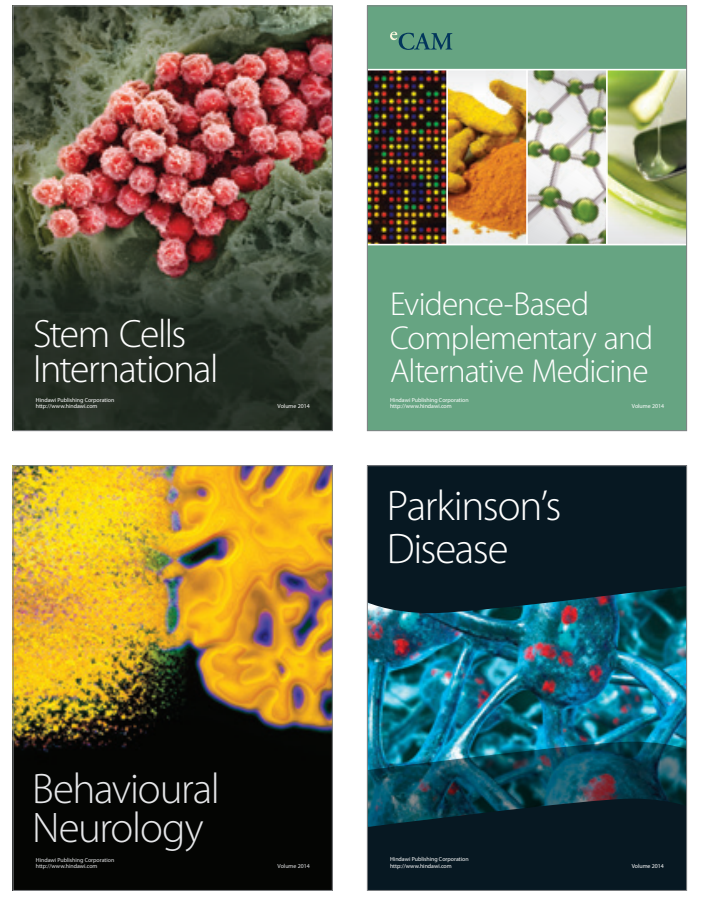
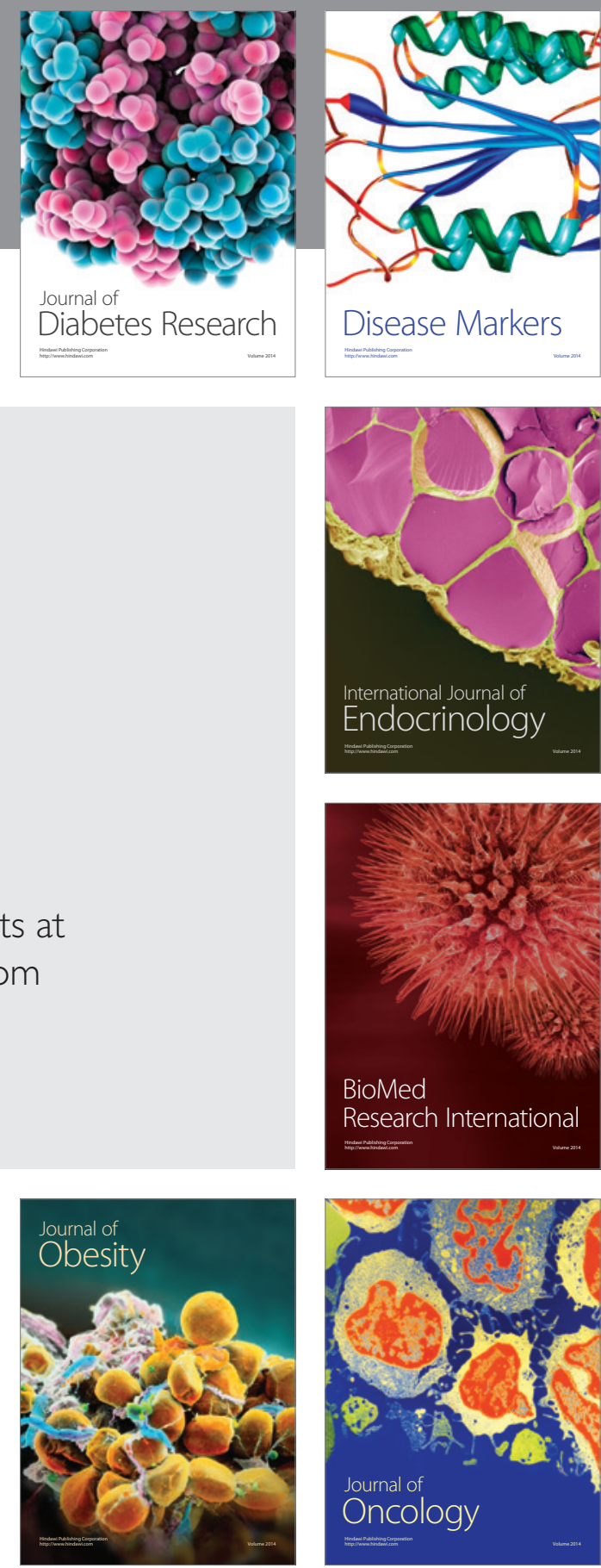

Disease Markers
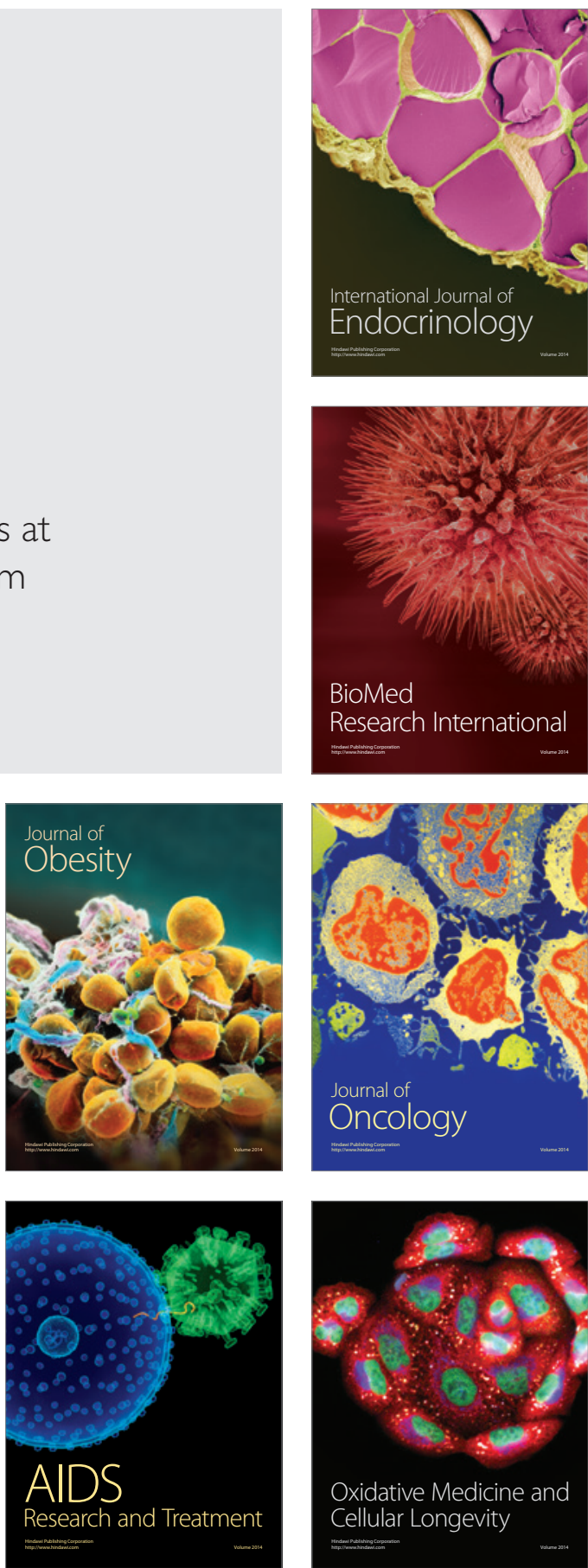